\title{
Exosomes as a Communication Tool Between the Lymphatic System and Bladder Cancer
}

\author{
Rebekah J. Park ${ }^{1}$, Yeo Jin Hong ${ }^{1}$, Yifan Wu ${ }^{1}$, Paul Myungchul Kim¹, Young-Kwon Hong ${ }^{1,2}$ \\ ${ }^{1}$ Department of Surgery, Keck School of Medicine, University of Southern California, Los Angeles, Los Angeles, CA, USA \\ ${ }^{2}$ Norris Comprehensive Cancer Center, Keck School of Medicine, University of Southern California, Los Angeles, CA, USA
}

To the editor,

The late Dr. Judah Folkman (February 24, 1933-January 14, 2008), a pioneer in the field of vascular biology and medicine, was the first to propose that growing tumors need a new network of blood vessels to survive and thrive, and that inhibition of the new blood vessel growth, termed angiogenesis, could suppress tumor growth and progression [1]. Since then, numerous antiangiogenic agents and new therapies have been developed and clinically evaluated to inhibit tumor growth $[2,3]$. Similarly, angiogenesis of lymphatic vessels, termed lymphangiogenesis, is the process of new lymphatic vessel formation and expansion [4]. The lymphatic system is a network of lymphatic vessels that carry the interstitial fluid, cells, lipids and other large molecules, collectively called lymph. Lymph contains lymphocytes and, thus, the lymph system plays an important role in our immune system. The lymphatic system also includes secondary lymphoid tissues, such as lymph nodes, the spleen, tonsils, Peyer's patches and mucosa-associated lymphoid tissue [4]. While the blood vascular system is a circular system, where the fluid (blood) leaves the heart and comes back to the same organ, the lymphatic system is a linear vascular system that starts as capillary lymphatic vessels, and eventually connects to the subclavian vein via the biggest caliber lymphatic vessels, called the thoracic duct. Although the blood and the lymphatic vessels share a number of similarities in their structure, anatomy, function, and more, the systems also have as many disparities in their pathophysiology.

Lymph node metastasis is considered to have a major prog- nostic value, boasting characteristics such as tumor aggression and a worse cancer survival rate. It is now generally accepted that lymphangiogenic factors and lymphangiogenesis promote metastasis to lymph node and distant organs [5]. Intra- and peritumoral lymphatic vessels are now hypothesized to play an important role in the interaction between lymphatic endothelial cells (LECs) and cancer cells, leading to cancer metastasis. Although the molecular and cellular mechanisms that control these processes have not yet been completely identified, the remodeling of tumor-associated lymphatics has been reported to provide important clinical information on metastasis and prognosis. In the case of metastatic melanomas with enlarged tumor-associated lymphatics, the lymphatic vessel area was significantly associated with poor disease-free and overall survival $[6,7]$. Thus, targeting lymphangiogenesis through inhibiting lymphangiogenic signaling pathways could be a potential tool for blocking or delaying metastasis. Both tumor cells and stromal cells secrete a number of cytokines, growth factors (such as vascular endothelial growth factors [VEGFs]), and other signaling molecules, such as exosomes, which promote lymphatic enlargement and remodeling. Therefore, cancer-associated lymphangiogenesis may well be a strong prognostic indicator for the risk of lymph node metastasis in various cancer types $[8,9]$.

We have previously reported 3 fluorescent lymphatic reporter animals ( 2 mouse lines and 1 rat model). Two transgenic mouse models, Prox1-EGFP [10] and Prox1-tdTomato [11], harbor bacterial artificial chromosome (BAC) where EGFP and tdTomato reporter genes were inserted respectively under the

Corresponding author: Young-Kwon Hong (iD https://orcid.org/0000-0001-8245-875X Departments of Surgery, University of Southern California, Norris Comprehensive Cancer Center, 1450 Biggy St. NRT6501, Los Angeles, CA 90033, USA E-mail: young.hong@usc.edu / Tel: +1-323-442-7825

This is an Open Access article distributed under the terms of the Creative Commons Attribution Non-Commercial License (http://creative-

Submitted: August 14, 2018 / Accepted after revision: September 13, 2018 

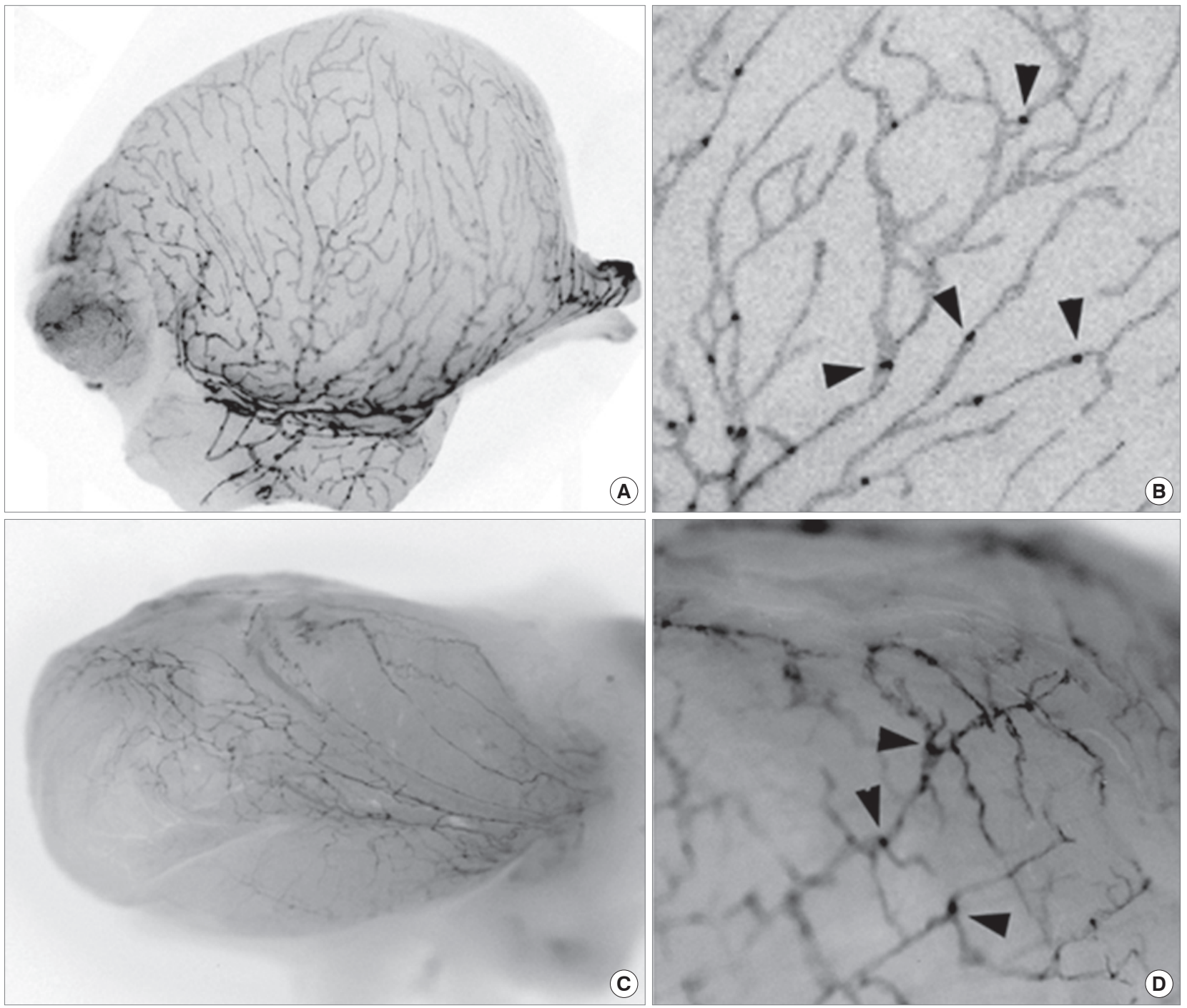

Fig. 1. Visualization of the bladder lymphatic networks using fluorescent lymphatic reporter mouse and rat. Bladders were isolated from adult Prox1-EGFP mouse (A, B) and rat (C, D), and subjected to fluorescent stereomicroscopy. Fluorescent signals were captured using a monochrome camera and shown in grayscale. (A, C) Gross images revealed a dense network of lymphatic vessels on the surface of the bladders. (B, D) Enlarged images demonstrate lymphatic vessels and sprouts along with luminal valves (arrowheads).

promoter of Proxl gene, which functions as the master regulator of lymphatic development. The fluorescent lymphatic reporter rat was generated using the mouse Prox1-EGFP BAC [12]. These reporter models have been very useful tools for the lymphatic researches, allowing direct visualization of the lymphatic networks, for example, on the surface of the bladder (Fig. 1).

Tumor-associated lymphatics drain the interstitial fluids, containing various signal molecules, cells, large molecules, and biological wastes, from tumors to the lymph nodes. Most cancer cells shed different types of microvesicles (e.g., exosomes) into the extracellular environment. These microvesicles, once consid- ered nonfunctional biological wastes, are now considered as important carriers of genetic and epigenetic information within the tumor microenvironment [13]. Among a variety of microvesicles that fit between the size range of 5-100 $\mathrm{nm}$ are exosomes, which have been considered as the ideal vehicle to play a role in lymphatic transport between cancer cells and the lymphatic system [14]. These nano-sized vesicles can be isolated from multiple biological fluids, including urine, plasma, pleural effusion, saliva, and lymphatic fluids $[15,16]$. Tumor-derived exosomes have been reported to contain nucleic acids (e.g., DNA, mRNA, miRNA, and small noncoding RNAs), proteins, and metabolites. 

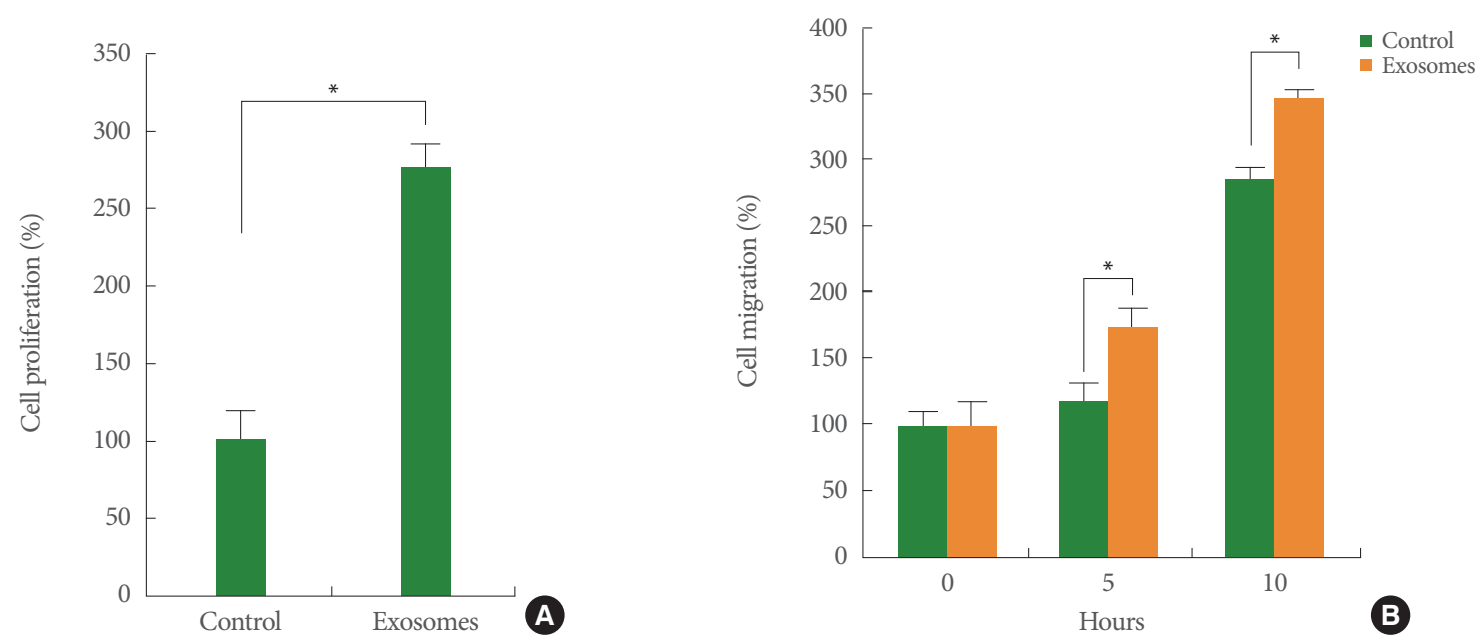

Fig. 2. Bladder cancer cell-derived exosomes enhance the proliferation and migration of cultured lymphatic endothelial cells (LECs). Conditioned medium was harvested from cultured T24 bladder cancer cells (American Type Culture Collection, ATCC) and subjected to the ultracentrifugation process to isolate tumor-secreted exosomes. Human dermal LECs (ATCC) were treated with the isolated exosomes or phosphate buffered saline (PBS) (control). Migration (A) and proliferation (B) was compared between PBS-treated LECs and exosome-treated LECs. ${ }^{*} \mathrm{P}<0.05$.

Since exosomes have specific membrane structures, those genetic materials can be protected from degradation in the harsh extracellular environment. Tumor-derived exosomes can activate stroma, promote cancer metastasis and chemo-resistance, and contribute to inflammatory responses, endothelial cell migration, and angiogenesis. In cancer-endothelial cell interactions, oncogenic exosomes might stimulate lymphangiogenesis and potentially enhance cancer cell invasion. Proteomics profiling of the cancer-derived exosomes showed that exosomes are enriched with proteins, whose functions are associated with cell adhesion, cytoskeletal remodeling, and signal transduction [17]. On the contrary, a recent study demonstrated that exosome-rich endothelial vesicles released from LECs accumulate in the perivascular stroma and guide the migration of dendritic cells [18]. This study provides an important implication that the LEC-secreted exosomes likely attract cancer cells and guide their metastasis.

Bladder cancer is the second most common genitourinary malignancy [19-21]. Compared to nonmuscle invasive bladder cancer (NMIBC), which is curable through surgical intervention, muscle invasive bladder cancer (MIBC) generally shows a worse prognosis [22-24]. Several recent studies suggest that lymphangiogenesis is associated with the muscle invasiveness of bladder cancers. The density of peritumoral lymphatics have been correlated with both regional metastasis and survival in bladder cancer $[25,26]$, and upregulation of a key lymphangiogenesis factor, VEGFC, was found in bladder cancers [27]. In addition, downregulation of BLACAT2 (bladder cancer-associated transcript 2), a long noncoding RNA, is positively associated with lymphangiogenesis and invasiveness [28]. Given these findings showing that lymphangiogenesis plays an important role in bladder cancer metastasis, it is necessary to determine the roles of lymphatic systems in the development of MIBC from NMIBC. In addition, tumor-derived exosomes may have a biological function in conditioning lymphangiogenesis within the bladder microenvironment. Our own data show that exosomes isolated from T24 bladder cancer cells enhance the proliferation and migration of cultured human LECs (Fig. 2), suggesting a potential regulatory mechanism of tumor-derived exosomes in lymphatic systems through the horizontal transfer of genetic and epigenetic information.

Despite, the casual link between exosomes and lymphatic metastasis of bladder cancer remains to be firmly established. For example, what specific bioactive materials are encapsulated or enriched in the tumor-derived exosomes that stimulate the lymphatics? And what signaling cascades are activated by exosome exposure in LECs? More studies are needed to advance our knowledge of the impact of the bladder lymphatics in metastatic bladder cancers and their exosomes, and of the identity and nature of tumor exosome contents. Similar questions could be also asked to lower urinary tract symptoms (LUTS) including urinary incontinence, overactive bladder, interstitial cystitis/ bladder pain syndrome, and urinary tract infections et al. How- 
ever, not much efforts have been invested to understand the casual connection between bladder lymphatic system and LUTS [29]. Thus, our collaborative research team has started working on the biological roles of urinary exosomes in LUTS and its underlying mechanism in bladder pathogenesis. We believe that profiling exosomes using multiomics approaches may shed much-needed light to these questions, and help us better understand the underlying mechanisms and biological link between exosomes and lymphatics in bladder diseases including bladder cancer as well as LUTS.

- Grant/Fund Support: This study was supported in part by National Institutes of Health (to YK Hong, R01DK114645).

- Research Ethics: The animal studies were approved by Institutional Animal Care and Use Committee (IACUC), University of Southern California (PI: YK Hong).

- Conflict of Interest: No potential conflict of interest relevant to this article was reported.

\section{REFERENCES}

1. Cao Y, Langer R. A review of Judah Folkman's remarkable achievements in biomedicine. Proc Natl Acad Sci U S A 2008;105:13203-5.

2. Kerbel R, Folkman J. Clinical translation of angiogenesis inhibitors. Nat Rev Cancer 2002;2:727-39.

3. Li T, Kang G, Wang T, Huang H. Tumor angiogenesis and anti-angiogenic gene therapy for cancer. Oncol Lett 2018;16:687-702.

4. Aspelund A, Robciuc MR, Karaman S, Makinen T, Alitalo K. Lymphatic system in cardiovascular medicine. Circ Res 2016;118:515-30.

5. Dellinger MT, Witte MH. Lymphangiogenesis, lymphatic systemomics, and cancer: context, advances and unanswered questions. Clin Exp Metastasis 2018 May 28 [Epub]. https://doi.org/10.1007/ s10585-018-9907-9.

6. Fankhauser M, Broggi MAS, Potin L, Bordry N, Jeanbart L, Lund AW, et al. Tumor lymphangiogenesis promotes $\mathrm{T}$ cell infiltration and potentiates immunotherapy in melanoma. Sci Transl Med 2017;9(407). pii: eaal4712. https://doi.org/10.1126/scitranslmed. aal4712.

7. Raica M, Jitariu AA, Cimpean AM. Lymphangiogenesis and antilymphangiogenesis in cutaneous melanoma. Anticancer Res 2016;36:4427-35.

8. Ran S, Volk L, Hall K, Flister MJ. Lymphangiogenesis and lymphatic metastasis in breast cancer. Pathophysiology 2010;17:229-51.

9. Lund AW, Duraes FV, Hirosue S, Raghavan VR, Nembrini C, Thomas $\mathrm{SN}$, et al. VEGF-C promotes immune tolerance in B16 melano- mas and cross-presentation of tumor antigen by lymph node lymphatics. Cell Rep 2012;1:191-9.

10. Choi I, Chung HK, Ramu S, Lee HN, Kim KE, Lee S, et al. Visualization of lymphatic vessels by Prox1-promoter directed GFP reporter in a bacterial artificial chromosome-based transgenic mouse. Blood 2011;117:362-5.

11. Hong M, Jung E, Yang S, Jung W, Seong YJ, Park E, et al. Efficient assessment of developmental, surgical and pathological lymphangiogenesis using a lymphatic reporter mouse and its embryonic stem cells. PLoS One 2016;11:e0157126.

12. Jung E, Gardner D, Choi D, Park E, Jin Seong Y, Yang S, et al. Development and characterization of a novel Prox1-EGFP lymphatic and Schlemm's canal reporter rat. Sci Rep 2017;7:5577.

13. Li X, Wang Y, Wang Q, Liu Y, Bao W, Wu S. Exosomes in cancer: small transporters with big functions. Cancer Lett 2018;435:55-65.

14. Xu R, Rai A, Chen M, Suwakulsiri W, Greening DW, Simpson RJ. Extracellular vesicles in cancer - implications for future improvements in cancer care. Nat Rev Clin Oncol 2018 May 23 [Epub]. https://doi. org/10.1038/s41571-018-0036-9.

15. Franzen CA, Blackwell RH, Foreman KE, Kuo PC, Flanigan RC, Gupta GN. Urinary exosomes: the potential for biomarker utility, intercellular signaling and therapeutics in urological malignancy. J Urol 2016;195:1331-9.

16. Nawaz M, Camussi G, Valadi H, Nazarenko I, Ekström K, Wang X, et al. The emerging role of extracellular vesicles as biomarkers for urogenital cancers. Nat Rev Urol 2014;11:688-701.

17. Carrasco-Ramírez P, Greening DW, Andrés G, Gopal SK, MartínVillar E, Renart J, et al. Podoplanin is a component of extracellular vesicles that reprograms cell-derived exosomal proteins and modulates lymphatic vessel formation. Oncotarget 2016;7:16070-89.

18. Brown M, Johnson LA, Leone DA, Majek P, Vaahtomeri K, Senfter $\mathrm{D}$, et al. Lymphatic exosomes promote dendritic cell migration along guidance cues. J Cell Biol 2018;217:2205-21.

19. Kiemeney LA, Witjes JA, Verbeek AL, Heijbroek RP, Debruyne FM. The clinical epidemiology of superficial bladder cancer. Dutch South-East Cooperative Urological Group. Br J Cancer 1993;67: 806-12.

20. Borden LS Jr, Clark PE, Hall MC. Bladder cancer. Curr Opin Oncol 2003;15:227-33.

21. Hall MC, Chang SS, Dalbagni G, Pruthi RS, Seigne JD, Skinner EC, et al. Guideline for the management of nonmuscle invasive bladder cancer (stages Ta, T1, and Tis): 2007 update. J Urol 2007;178:231430.

22. Kamat AM. Commentary on "Surveillance guidelines based on recurrence patterns after radical cystectomy for bladder cancer: the 
Canadian Bladder Cancer Network experience." Yafi FA, Aprikian AG, Fradet Y, Chin JL, Izawa J, Rendon R, Estey E, Fairey A, Cagiannos I, Lacombe L, Lattouf JB, Bell D, Saad F, Drachenberg D, Kassouf W. Department of Surgery (Urology), McGill University, Quebec, Canada: BJU Int 2012;110(9):1317-23 [Epub 2012 Apr 13]. Urol Oncol 2013;31:717-8.

23. Large MC, Cohn JA, Steinberg GD. Optimal risk-adapted surveillance strategies for NMIBC, including upper tract imaging. Urol Clin North Am 2013;40:305-15.

24. Black PC, Dinney CP. Growth factors and receptors as prognostic markers in urothelial carcinoma. Curr Urol Rep 2008;9:55-61.

25. Fernández MI, Bolenz C, Trojan L, Steidler A, Weiss C, Alken P, et al. Prognostic implications of lymphangiogenesis in muscle-invasive transitional cell carcinoma of the bladder. Eur Urol 2008;53: 571-8.
26. Zhou M, He L, Zu X, Zhang H, Zeng H, Qi L. Lymphatic vessel density as a predictor of lymph node metastasis and its relationship with prognosis in urothelial carcinoma of the bladder. BJU Int 2011;107:1930-5.

27. Keck B, Wach S, Taubert H, Zeiler S, Ott OJ, Kunath F, et al. Neuropilin-2 and its ligand VEGF-C predict treatment response after transurethral resection and radiochemotherapy in bladder cancer patients. Int J Cancer 2015;136:443-51.

28. He W, Zhong G, Jiang N, Wang B, Fan X, Chen C, et al. Long noncoding RNA BLACAT2 promotes bladder cancer-associated lymphangiogenesis and lymphatic metastasis. J Clin Invest 2018;128: 861-75.

29. Urinology Think Tank Writing Group. Urine: Waste product or biologically active tissue? Neurourol Urodyn 2018;37:1162-8. 